Learning \& Verbai Beńavior, $1967,6,328-334$

PRIMOFF, E. Backward and forvard association as an organizing act in serial and in paired associate learning. Joumal of Psychology, $1938,5,375-395$.

THORNDIKE, E. L., \& LORGE, I. The teacher's word book of 30,000 words. New York: Columbia University, 1944.

WEAVER, G. E., HOPKINS, R, H., \& SCHULZ, R. W. The $A-B, B-C, A-C$ mediation paradigm:
A-C performance in the absence of study trials. Journal of Experimental Psychology, 1968 , $77,670-675$

YOLNG, R. K. A comparison of two me thods of learning serial associations. American Joumal of Psychology, 1959, 72, 554-559.

YOUNG, R. K. Paired-associate learning when the same items occur as stimuli and responses. Journal of Experimental Psychology, 1961, $61,315-318$.

\section{Effects of proportion of positive instances and degree of restriction on the induction of a principle*}

\author{
DENNIS DERVIN and KENNETH DEFFENBACHER† \\ University of Nebraska at Omaha, Omaha, Nebr. 68101
}

Ss attempted to discover a principle by which numbers and letters were paired. Seven groups of Ss varied on two dimensions, proportion of positive instances sampled and degree of restriction in selecting instances for test. Ss who sampled a higher proportion of positive instances were superior to those sampling a lower proportion. Ss who were unrestricted in their selection of instances, because they sampled a higher proportion of positive instances, performed better than Ss who were restricted and sampled a lower proportion. Finally, when both restricted and unrestricted groups were equated for proportion of positive instances sampled, performance was similar.

A number of studies have investigated the effects of the degree of restriction placed upon $\mathrm{S}$ in his attempt to discover the solution to a problem (Huttenlocher, 1962; Duncan, 1964; Hunt, 1965; Murray \& Gregg, 1969). Both the Huttenlocher and Murray and Gregg studies utilized single-cue concept problems, which $S$ solved either under a selection (unrestricted in instance selection) or a reception (restricted in instance selection) paradigm. Each study found that the reception group solved the problems more rapidly than did the selection group. Hunt's Ss solved considerably more difficult problems than those of the previous two studies; Ss had to solve either conjunctive concept problems or to learn to recognize legitimate letter strings generated by a finite-state grammar. Hunt found that Ss solving under selection conditions were superior to those solving under reception conditions. The results of

*This research was carried out by the senior author at the University of Nebraska at Omaha in partial fulfillment of the requirements for the MA degree.

† Requests for reprints should be sent to Kenneth Deffenbacher, Department of Psychology, Lniversity of Nebraska at Omaha, Omaha, Nebraska 68101 the three studies discussed thus far would seem to suggest that freedom in instance selection is advantageous only when the task is a difficult one. Duncan's study does not appear to fit this pattern. He found the same result as did Hunt, yet his task would appear to be less difficult; Ss had to discover E's principle for pairing numbers and letters.

There may be a confounding factor in Duncan's study, however. Ss who were unrestricted in instance selection may have been sampling a higher proportion of positive instances than were $S s$ in the partially and totally restricted groups. Duncan arbitrarily imposed a 50:50 split of positive and negative instances on $\mathrm{Ss}$ in the partially and totally restricted groups. As Ss in the unrestricted group were free to choose any instance they wished, their proportion of positive instances could thus deviate significantly from the 0.50 proportion imposed on the other groups. Hovland \& Weiss (1953) and Haygood \& Stevenson (1967) have presented evidence showing that naive Ss utilize positive instances much more efficiently than they do negative instances.

The present study seeks to replicate Duncan's study and then to show that his unrestricted group's greater problem-solving efficacy was due, not to the restriction variable, but rather to the higher proportion of positive instances that they sampled.

Seventy Ss were drawn from an introductory psychology course at the University of Nebraska at Omaha as a part of a course requirement. Ten Ss were unsystematically assigned to each of seven treatment groups.

In order to test the contention that Duncan's results were at least partially an artifact of the proportion of positive instances sampled (the unrestricted group sampling a higher proportion than the restricted groups), it was necessary to run the unrestricted group (U group) first. This was necessary in order to determine the mean proportion of positive instances sampled by this group.

Of the seven groups, four were replicates of Duncan's conditions. These four groups included the $U$ group, two partially restricted groups, and one totally restricted group. In Group RN (restricted numbers), $S$ was allowed to choose any letter he wished but was told which number to write down. Similarly, Group RL (restricted letters) chose any number but was told which letter to record. Group RNL (restricted numbers and letters) was told which number and which letter to record. All three groups (RN, RL, and RNL) had the same 50:50 split of positive and negative instances imposed on them, as was the case in Duncan's study. These groups are referred to, respectively, as RN50, RL50, and RNL50.

The remaining three groups operated under the same conditions as those stated above. However, these groups sampled the same proportion of positive instances as that sampled by the $\mathrm{U}$ group. They are referred to as RN (U\%), RL (U\%), and RNL (U\%). These groups were added to test the contention that Duncan's results were due, in part at least, to the proportion of positive instances sampled.

The task was the same as in Duncan's study. By creating number-letter pairs. $S$ attempted to discover the principle: "Any of the even numbers $(2,4,6,8)$ may be paired with any of the letters A.E; any of the odd numbers $(1,3,5,7)$ may be paired with any of the letters F-J." Each trial consisted of the creation of a number-letter pair, followed by feedback from $E$ as to whether the instance was or was not an example of the principle. $\mathrm{S}$ was allowed to work at his own pace and was allowed to guess at the principle at any time. After each guess, $E$ informed $S$ whether the hypothesis was correct, partially correct, or wrong. If $S$ had not stated the principle correctly after $25 \mathrm{~min}$, he was stopped and 
recorded as a nonsolver.

The only materials required for this experiment were sheets on which $S$ recorded his choices. There were two forms of this sheet. One form had the numbers 1.8 printed horizontally in normal order in the upper left comer and the letters A-J printed in the upper right. There were 80 blanks on the sheet in four columns of 20 each. Groups $U, R N$, and $R L$ used this form. The other form was the same as the first, except that one of the numbers 1.8 was printed in each blank. Within any block of eight trials, each number was used once, with random assignment of numbers within blocks. Group RNL used this form.

\section{RESULTS}

The mean proportion of positive instances sampled by Group $U$ was 0.70 . Accordingly, the experimental conditions of the three restricted groups added in this study-RN70, RL70, and RNL70-were such that within a block of 40 trials, 0.70 of the instances were positive. It is not likely that $\mathrm{Ss}$ in these three groups who solved in less than 40 trials actually sampled a proportion other than 0.70 ; 2 tests for the difference between observed and predicted proportions yielded no significant differences for any groups. In addition, the proportions of nonsolvers in the groups replicating Duncan's study were virtually identical to those obtained by Duncan. The proportion of nonsolvers in each of the groups added in the present study was 0.10 .

The data obtained was analyzed by nonparametric techniques, as there was truncation imposed by the 25 -min solution time requirement. The median number of number-letter pairs to solution were: Group $U=34.0$, Group $R N 50=54.5$, Group RL50 $=47.0$, Group RNL50 = 51.0, Group RN70 $=20.0$, Group RL70 = 33.0, Group $R N L 70=34.0$. A Kruskal-Wallis analysis of variance by ranks on this data yielded a significant treatment effect, $\mathrm{H}(6)=55.70, \mathrm{p}<.001$. In order to isolate differences among groups, separate Kruskal-Wallis tests were applied to groups involved in each of three sets of planned comparisons. An analysis involving Groups $\mathrm{U}, \mathrm{RN70}, \mathrm{RL70}$, and RNL70 produced $H(3)=6.58, p<.10$. Finally, analysis of Groups RN50, RL50, RNL50, RN70, $H(3)=6.58, p<.10$. Finally, analysis of Groups RN50, RL50, RN70, RL70, and RNL70 yielded $H(5)=22.0, p<.001$.

Specific comparisons were then conducted using the Mann-Whitney $U$ test. Three comparisons were made in the first set of groups. The difference between $\mathrm{U}$ and RN50 yielded $\mathrm{U}(6,9)=9.0, \mathrm{p}<.025$. The $U$ vs RL50 comparison produced $\mathrm{U}(7,9)=12.0, p=.025$. Finally, the difference between $U$ and RNL50 yielded $\mathrm{U}(7,9)=8.0, \mathrm{p}<.01$.

Three comparisons were also made in the third set of groups. The comparison between RN50 and RN70 yielded $\mathrm{U}(6,9)=2.0, \mathrm{p}=.001$. The RL50 vs RL70 comparison produced $\mathrm{U}(7,9)=9.5$, $\mathrm{p}<.025$. Finally, the difference between RNL50 and RNL70 yielded $\mathrm{U}(7,9)=8.5$, $\mathrm{p}<.01$.

\section{DISCUSSION}

The present study replicated the results obtained by Duncan in that the $U$ group was found to be superior to those restricted groups sampling a 0.50 proportion of positive instances. Furthermore, those restricted groups sampling a $\mathbf{0 . 7 0}$ proportion of positive instances were superior to the 0.50 -proportion groups. The attempt to demonstrate differences among the unrestricted and restricted groups matched for proportion of positive instances sampled failed.

On the basis of the results of the present study, it would appear reasonable to conclude that the efiects obtained by Duncan were confounded by the operation of the varying proportion of positive instances sampled by his groups. Moreover, the nonsignificant Kruskal-Wallis analysis of Groups U, RN70, RL70, and RNL70 would seem to suggest that the restriction variable did not account for much variance in Duncan's study.

Two additional comments should be made. Given the results of the present study, Duncan's study would now appear to fit the pattern of results found by other investigators of the restriction variable. The pattern that emerges is that restriction is advantageous for simple tasks (Huttenlocher, 1962; Murray \& Gregg, 1969); complete lack of restriction is advantageous for complex tasks (Hunt, 1965); and the restriction variable is not an important one for tasks of intermediate difficulty such as Duncan's. Finally, any future research using a restriction variable should make every effort to equate treatment groups for proportion of positive instances sampled.

\section{REFERENCES}

DUNCAN, C. P. Induction of a principle. Quarterly Journal of Experimental Psychology, 1964, 16, 373-377.

HAYGOOD, R. C., \& STEVENSON, M. Effects of proportion of positive instances on concept learning. Psychological Reports, 1967, 20, 179-182.

HOVLAND, C. I., \& WEISS, W. Ttansmission of information concerning concepts through positive and negative instances. Journal of Experimental Psychology, 1953, 45, 175-182.

HUNT, E. B. Selection and reception conditions in grammar and concept learning. Journal of Verbal Learning \& Verbal Behavior, 1965, 4, 211-215.

HUTTENLOCHER, J. Effects of manipulation of attributes on efficiency of concept formation. Psychological Reports, 1962, 10, 503-509.

MURRAY, F. S., \& GREGG, R. E. Reception versus selection procedures in concept learning. Journal of Experimental Psychology, $1969,82,571.572$. 